\title{
Mortalidade por causas relacionadas ao aborto no Brasil: declínio e desigualdades espaciais ${ }^{1}$
}

\author{
Bruno Gil de Carvalho Lima²
}

\begin{abstract}
RESUMO Além de constituir causa freqüente de internamentos obstétricos em países pobres, o aborto representa a incapacidade do sistema público de saúde de prover informação suficiente sobre métodos contraceptivos para prevenir gestações em vez de interrompê-las. No Brasil, as altas taxas de utilização de serviços de saúde por abortamentos refletem as dificuldades persistentes de contracepção e planejamento familiar. Além disso, a mortalidade por aborto serve como indicador da qualidade dos procedimentos abortivos, um ponto importante num país onde tal prática é ilegal e, portanto, clandestinamente realizada. No presente estudo, analisamos as taxas de mortalidade por causas relacionadas ao aborto entre mulheres de 10 a 54 anos de idade, incluindo aquelas que morreram por abortamentos espontâneos e provocados, de 1980 a 1995, segundo região de residência. As informações utilizadas foram obtidas do banco de dados sobre mortalidade do Sistema Único de Saúde - Ministério da Saúde. Dados sobre população foram obtidos junto à Fundação Instituto Brasileiro de Geografia e Estatística. Estudaram-se 2602 óbitos. Do total de óbitos, $15 \%$ foram devidos a aborto retido, aborto espontâneo e aborto induzido com indicação legalmente admitida. Oitenta e cinco por cento dos óbitos foram causados por aborto induzido sem indicação legalmente admitida e por aborto sem causa especificada. Os coeficientes de mortalidade por causas relacionadas ao aborto têm decrescido continuamente no Brasil, mas tais avanços têm-se distribuído desigualmente no país. A região que apresentou a menor queda na taxa (38\% em 15 anos) foi o Nordeste. As mulheres que morreram por aborto tiveram uma média de idade decrescente no período estudado.
\end{abstract}

O planejamento familiar não constitui questão simples em lugar algum. Contudo, nos países em desenvolvimento, as dificuldades quanto à decisão sobre o tamanho da prole e sobre os métodos para prevenir uma gravi-

\footnotetext{
1 Apresentado com o título "Mortality by abortionrelated causes in Brazil: decline and spatial inequalities" no $10^{\circ}$ Congresso Mundial de Reprodução Humana, Salvador, BA, Brasil, 4 a 8 de maio de 1999.

2 Universidade Federal da Bahia, Faculdade de Medicina. Correspondência e pedidos de separatas devem ser enviados para o seguinte endereço: Rua Padre Manoel Barbosa 27, Apto. 502, Itaigara, CEP 41815-050, Salvador, BA, Brasil. E-mail: brunogil@ doctor.com
}

dez indesejada são, certamente, de mais difícil resolução, e trazem conseqüências mais sérias sobre os problemas sociais já existentes do que nas nações industrializadas (1-6).

Devido a essas dificuldades, a prática da indução de abortamentos tem aumentado em muitos países pobres. De fato, o cuidado médico prestado a pacientes submetidas a abortos vem mostrando uma tendência a ultrapassar a assistência ao parto em algumas regiões (7). Mesmo sendo proibida, com punição prevista por lei, a interrupção da gravidez (em vez da sua prevenção) tem sido um importante método de controle da natalidade para milhares de pessoas na América Latina $(2,5,8,9)$. Recomendações da Organização das Nações Unidas (ONU) (10), assim como medidas governamentais visando decidir unilateralmente sobre natalidade e obstáculos legais ao aborto, não têm conseguido evitar que muitas mulheres procurem aborteiros clandestinos que nem sempre estão preparados para prover serviços adequados, transformando o que poderiam ser procedimentos simples e de baixo risco em intervenções perigosas, potencialmente danosas e letais $(8,11,12)$. 
O aborto é, portanto, um problema social e político, com envolvimento de aspectos econômicos, educacionais e religiosos, mas que freqüentemente se apresenta como uma questão do setor da saúde (13-17). É surpreendente a demanda por abortamentos no Brasil, a despeito de sua ilegalidade e do fato de a opinião dominante na sociedade lhe ser contrária - principalmente nas classes sociais mais baixas, com piores níveis educacionais (18). É clara a necessidade de discutir o tema de forma a respeitar a opinião feminina (19) e a resolver os problemas implicados na gênese dessa demanda, uma vez que até mesmo os profissionais de saúde já se mostraram incapacitados para a tomada de decisões apropriadas e para oferecer orientação e assistência na escolha da melhor solução para as gestações indesejadas (20).

$\mathrm{O}$ estudo da mortalidade materna traz, por si só, certas dificuldades, devido ao problema bastante disseminado do sub-registro de óbitos nas estatísticas oficiais, uma deficiência que ocorre mesmo em países desenvolvidos. ${ }^{3}$ Mais problemática ainda é a notificação do aborto e de alguns problemas de saúde a ele relacionados.

No presente trabalho, optou-se por estudar a mortalidade por causas relacionadas ao aborto. Estudos de morbidade, os quais compõem a maioria daqueles já desenvolvidos sobre o assunto, teriam dificuldades ainda maiores para comparar os resultados dos diversos estados brasileiros. Além disso, o sistema de informações de mortalidade do Ministério da Saúde fornece dados razoavelmente confiáveis, embora ainda apresentem problemas, sobretudo erros na seleção da causa básica da morte e um grande número de óbitos por "sinais e sintomas de afecções mal definidas" (grupo XVI, causas 870 a 799 da nona revisão da Classificação Internacional do Doenças, CID-9). Assim, o objetivo do presente trabalho foi comparar a evolução dos coeficientes de mortalidade por aborto

\footnotetext{
Laurenti R. Usos das estatísticas de mortalidade. Apresentado no $2^{\circ}$ Encontro Nacional do SubSistema de Informações de Mortalidade, Brasília, setembro de 1990. [documento mimeografado].
}

entre as regiões brasileiras, a fim de formular hipóteses que possam explicar as desigualdades espaciais dos coeficientes.

\section{MATERIAIS E MÉTODOS}

A mortalidade por causas relacionadas ao aborto no Brasil foi estudada através dos coeficientes de mortalidade em mulheres de 10 a 54 anos de idade, de 1980 a 1995 . Foram incluídos os óbitos pelas causas 632 (aborto retido) e 634 a 637 (aborto espontâneo, aborto induzido com indicação legalmente admitida, aborto induzido sem indicação legalmente admitida e aborto por causa não especificada, respectivamente) da CID-9. Os dados acerca do número de mortes foram coletados no sistema de informações de mortalidade do Sistema Único de Saúde, Ministério da Saúde (SIM-DATASUS-MS), por meio do programa TABX fornecido pelo DATASUS. Foram estudados 2602 óbitos, classificados por unidade da federação, ano de ocorrência e idade da mulher. Deu-se maior atenção aos óbitos por abortamento praticado sem indicação legalmente prevista ou por aborto não especificado.

Informações sobre o tamanho da população foram obtidas junto à Fundação Instituto Brasileiro de Geografia e Estatística (IBGE). A população em 1980, 1991 e 1996 foi retirada dos censos populacionais de 1980 e 1991 e da contagem populacional realizada em 1996. O método de interpolação geométrica foi usado para estimar a população nos anos intercensitários. O número de mulheres entre 10 e 54 anos em cada estado e ano foi usado como denominador das taxas calculadas.

Os coeficientes de mortalidade por aborto em cada estado demonstraram uma tendência a variar bastante ano a ano, tornando mais adequada a análise por região. Assim sendo, os estados foram agrupados nas cinco macrorregiões geográficas (Norte, Nordeste, Sudeste, Sul e Centro-Oeste) e as taxas, recalculadas. Na tentativa de reduzir a oscilação dos coeficientes, que persistiu até certo ponto após agrupados os estados, os anos também foram reuni- dos, em quadriênios (1980 a 1983, 1984 a 1987, 1988 a 1991 e 1992 a 1995).

A mortalidade por faixa etária também foi estudada. Nessa etapa, os óbitos de todo o país foram analisados em conjunto e apenas os anos de 1980 e 1995 foram incluídos, a fim de avaliar a tendência temporal da mortalidade relacionada ao aborto por grupos de idade no Brasil.

Testes de hipóteses não foram empregados neste estudo devido ao seu desenho descritivo, o que tornou desnecessária a análise de significância estatística.

\section{RESULTADOS}

Malgrado algumas variações anuais de grande amplitude (figura 1), todas as regiões apresentaram coeficientes com tendências decrescentes no período estudado. A mortalidade por causas relacionadas ao aborto no Brasil, em 1980, foi de 5,58 por 1000000 de mulheres (10 a 54 anos de idade), decaindo para 2,47 por 1000000 em 1995.

Do total de óbitos por aborto no Brasil nos anos analisados, $15 \%$ foram devidos às causas classificadas na CID-9 com os códigos 632 (aborto retido), 634 (aborto espontâneo) e 635 (aborto induzido com indicação legalmente admitida). $\mathrm{O}$ coeficiente de mortalidade por essas causas variou de 0,41 por 1000000 , em 1980, para 0,52 por 1000 000, em 1995.

Já os coeficientes de mortalidade pelas causas 636 (aborto induzido sem indicação legalmente admitida) e 637 (aborto - não especificado) foram bem maiores - 5,17 por 1000000 de mulheres de 10 a 54 anos, em 1980, e 1,94 por 1000000 , em 1995. Embora não se possa afirmar que todos os abortos sem especificação registrados no SIM tenham sido provocados, nem que o tenham sido ilegalmente, decidimos analisá-los em conjunto com a causa 636. Acreditamos que os abortos registrados como não especificados, que representaram $52 \%$ do total de mortes, não teriam sido classificados nessa categoria caso fossem espontâneos ou provocados com respaldo legal. A tabela 1 mostra a evolução das 
FIGURA 1. Evolução anual da mortalidade (em 1000000 de mulheres) por abortos induzidos sem indicação legalmente admitida ou não especificados, Brasil, 1980 a 1995

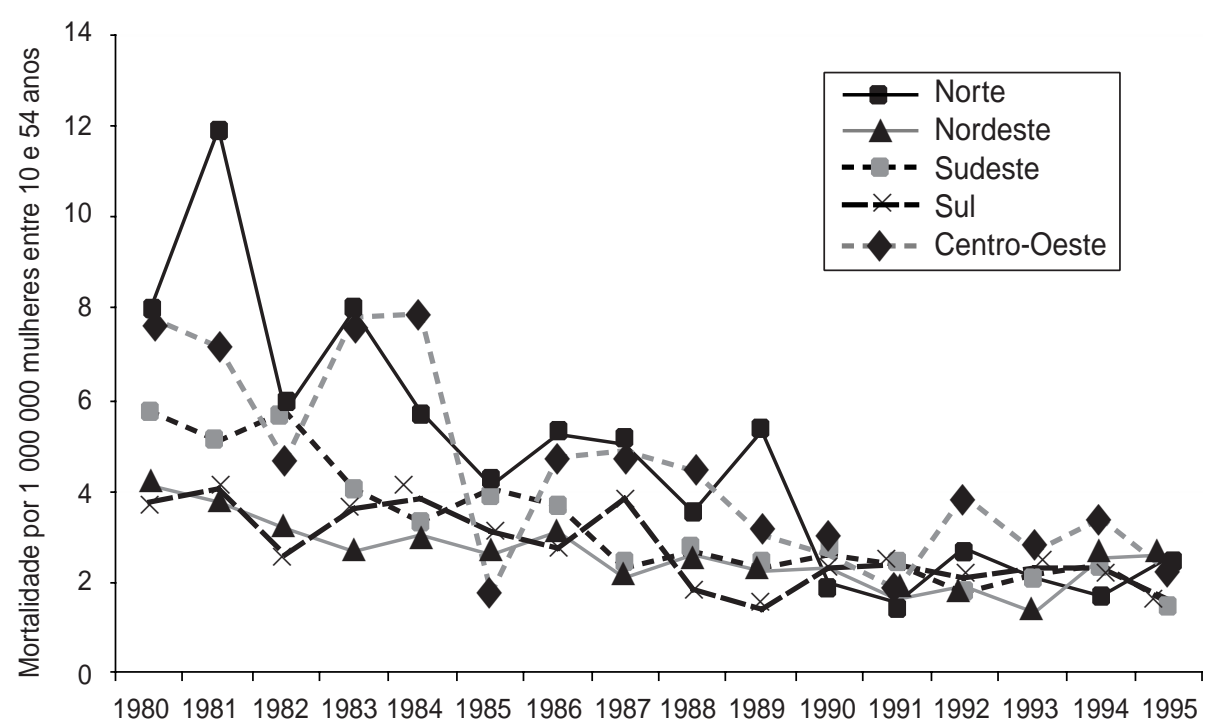

taxas de mortalidade pelas causas 636 e 637 em cada região do país. Exceções à tendência predominante ao declínio foram pequenos aumentos autolimitados dos coeficientes, mas que não chegaram a interferir com o perfil geral do período (figura 1).

A figura 1 fornece informações sobre as variações anuais da mortalidade por aborto ilegalmente induzido e não especificado nas regiões do Brasil. Esta figura mostra que houve uma aproximação dos coeficientes das diversas partes da federação.

Os dados da tabela 2 chamam a atenção para a progressiva concentração de óbitos pelas causas 636 e 637 entre as idades de 20 a 39 anos. Estes dados sugerem, ainda, um decréscimo na

TABELA 1. Evolução da mortalidade a por abortos induzidos sem indicação legalmente admitida ou não especificados, Brasil, 1980 a 1995

\begin{tabular}{lcccc}
\hline & \multicolumn{4}{c}{ Quadriênios } \\
\cline { 2 - 5 } Regiões & $1980-1983$ & $1984-1987$ & $1988-1991$ & $1992-1995$ \\
\hline Norte & 3,3 & 2,0 & 1,2 & 0,9 \\
Nordeste & 1,3 & 1,1 & 0,9 & 0,8 \\
Sudeste & 2,0 & 1,3 & 1,0 & 0,8 \\
Sul & 1,4 & 1,3 & 0,8 & 0,8 \\
Centro-Oeste & 2,7 & 1,9 & 1,2 & 1,2 \\
\hline
\end{tabular}

${ }^{a}$ Coeficientes de mortalidade por 1000000 mulheres de 10 a 54 anos, calculados dividindo-se o total de óbitos no quadriênio pela população média no período.
A tendência decrescente da mortalidade por aborto no Brasil é muito auspiciosa, apesar de vários dos fatores possivelmente implicados não indicarem necessariamente uma situação de saúde melhor no país. Informações recentes da literatura falam a favor de um aumento no número de procedimentos abortivos no Brasil nos últimos anos $(7,21)$. Além disso, o sistema governamental de autorizações de internação hospitalar (AIH-DATASUS-MS) disponibiliza taxas de utilização de leitos hospitalares que se contrapõem a qualquer hipótese de declínio de tal prática no país. A queda nos coeficientes de mortalidade poderia, pois, ser conseqüência da melhoria das condições técnicas de realização de abortamentos, reduzindo a letalidade. Isso, entretanto, não é provável, uma vez que o aborto continua sendo um crime em todas as regiões brasileiras e é praticado clandestinamente. Além disso, avanços na qualidade do procedimento de aborto teriam certamente contribuído para uma diminuição das taxas de hospitalização, a qual geralmente ocorre devido a complicações secundárias à inépcia dos que executam o aborto ilegalmente e à falta de estrutura e equipamentos adequados. Se alguma espécie de melhoria da qualidade ocorreu, isso deve ter se dado quanto ao cuidado médico prestado às mulheres que apresentam complicações pós-abortamento nas instituições de saúde disponíveis.

Um método relativamente novo de provocar um aborto, o uso de misoprostol, tem sido cada vez mais empregado no Brasil. Esse método químico, baseado na estimulação uterina por análogos de prostaglandina, inicialmente desenvolvido para o tratamento de doença dispéptica, também pode ter contribuído para a queda na mortalidade, por ser menos deletério e potencialmente menos perigoso, já que apresenta menor risco de infecção, perfuração de órgãos e hemorragia do que os métodos mecânicos predominantemente usados até pouco tempo atrás (5). Contudo, também o misoprostol está associado a conseqüências danosas, como malformações congêni$\operatorname{tas}(22)$. 
TABELA 2. Mortalidade por abortos induzidos sem indicação legalmente admitida ou não especificados conforme faixa etária, Brasil, 1980 e 1995

\begin{tabular}{ccrrr}
\hline Faixa etária (anos) & $1980^{\mathrm{a}}$ & $\%^{\mathrm{b}}$ & $1995^{\mathrm{a}}$ & $\%^{\mathrm{b}}$ \\
\hline 10 a 14 & 0,1 & 0,5 & 0 & 0 \\
15 a 19 & 2,3 & 8 & 1,8 & 13 \\
20 a 24 & 8,4 & 24 & 3,7 & 24 \\
25 a 29 & 8,7 & 21 & 4,0 & 24 \\
30 a 34 & 10,5 & 20 & 4,3 & 24 \\
35 a 39 & 10,8 & 7 & 2,3 & 12 \\
40 a 44 & 4,9 & 2 & 0,7 & 3 \\
45 a 49 & 1,7 & 0 & 0 & 0 \\
50 a 54 & 0 & 100 & 1,9 & 100 \\
Total & 5,2 & & & 0 \\
\hline
\end{tabular}

a Mortalidade por 1000000 mulheres na faixa etária.

b Percentual dos óbitos totais por abortos induzidos sem indicação legalmente admitida ou não especificados no ano, para cada faixa etária.

Uma piora na qualidade da notificação de óbitos também poderia ter provocado um comportamento como o apresentado nos resultados. Porém, tal explicação é improvável, pois o SUS tem conseguido avanços no campo de informações em saúde. Uma possibilidade que não pode ser desprezada é a de que os médicos ainda têm dificuldades na seleção da causa básica dos óbitos, particularmente em caso de doenças relacionadas a práticas ilegais, o que, sem dúvida, implica em certos obstáculos à coleta de uma anamnese (23). A grande parcela de casos classificados como aborto não especificado reafirma essa hipótese.

A progressão desigual dos coeficientes de uma região para outra apresentou um padrão curioso, para o qual não se pôde formular uma explicação lógica (tabela 1 e figura 1). Regiões com estruturas sociais completamente diferentes e situações econômicas díspares tiveram tendências temporais semelhantes, como as regiões Norte e Sudeste e Nordeste e Sul. Outro achado singular foi o fato de a região Centro-Oeste ter apresentado os melhores avanços no decréscimo das taxas, o que indubitavelmente não encontra paralelo nos demais indicadores de saúde ou educação dos estados daquela região.

Um ponto que deve ser destacado é que os estados nordestinos foram os que menos diminuíram seus coeficientes durante os 16 anos estudados (queda de $38 \%$ ). Isso pode ser atribuído às más condições das estruturas de saúde da região, mais um aspecto das deficiências enfrentadas pelo povo dessa que é uma das áreas mais pobres do país. Outro fator que pode haver influenciado nesse sentido é a possibilidade de que a região Nordeste apresentasse previamente níveis piores de sub-registro que as demais, devido à precariedade das estatísticas ou como resultado de singularidades ideoló- gicas regionais, como um arraigado machismo. Opiniões culturalmente influenciadas de médicos nordestinos podem ter tido algum papel na piora da classificação de causas de mortes em tais estados.

Característica interessante dos resultados encontrados foi o fato de as mulheres que morreram por aborto em 1995 serem, em média, mais jovens do que em 1980, indicando uma situação que já havia sido detectada anteriormente: adolescentes nulíparas são mais propensas a recorrer a um abortamento para evitar uma criança indesejada do que mulheres mais velhas, as quais tendem a decidir por um aborto quando já têm um ou mais filhos (7). Não surpreende que a idade progressivamente mais precoce de iniciação sexual no país (que também contribuiu, por exemplo, para o aumento da prevalência de infecção pelo vírus do papiloma humano e câncer cervical) tenha trazido mais uma razão para o aumento da participação percentual da faixa etária de 15 a 24 anos no total da mortalidade por aborto.

O problema do aborto continuará sendo um item importante na pauta de todas as nações, sobretudo aquelas em desenvolvimento. Decidir apenas pela sua legalização ou não, ou em que situações ele deve ser permitido, não resolverá a questão, pelo menos até que o nível educacional geral (e não somente o grau de informação sobre métodos contraceptivos e planejamento familiar) das populações melhore, ou até que modos mais apropriados de prevenção de gestações possam ser amplamente utilizados (24).

\section{REFERÊNCIAS}

1. Antrobus ACK. Temas contemporáneos en la atención de la salud maternoinfantil. Bol Oficina Sanit Panam 1986;100(4):387-399.

2. Robles RR, Martínez RE, Vera M, Alegría M. Factores socioculturales asociados con el uso de contraceptivos en Puerto Rico. Bol Oficina Sanit Panam 1988;104(1):23-33.

3. Meira AR, Ferraz FRC. Liberação do aborto: opinião de estudantes de Medicina e de Di- reito, São Paulo, Brasil. Rev Saude Publica 1989;23(6):465-472.

4. Costa E. A propriedade coletiva do feto na Romênia e a hipocrisia individualista no Brasil. Cadernos Saude Publica 1990;6(1):90-91.

5. Bailey PE, Llano Saavedra L, Kushner L, Welsh M, Janowitz B. Estudio hospitalario del aborto ilegal en Bolivia. Bol Oficina Sanit Panam 1988;104(2):144-159.
6. Puffer RR. La planificación familiar y la mortalidad materna e infantil en los Estados Unidos. Bol Oficina Sanit Panam 1993;115(5):389-404.

7. Schor N. Investigação sobre ocorrência de aborto em pacientes de hospital do centro urbano do Estado de São Paulo, Brasil. Rev Saude Publica 1990;24(2):144-151.

8. Fonseca W, Misago C, Correia LL, Parente JAM, Oliveira FC. Determinantes do aborto 
provocado entre mulheres admitidas em hospitais em localidade da região Nordeste do Brasil. Rev Saude Publica 1996;30(1):13-18.

9. Brasil. Código Penal: organização dos textos, notas remissivas e índices por Juarez de Oliveira. 34a ed. São Paulo: Saraiva; 1996. p. 73-74.

10. Hardy E, Rebello I. La discusión sobre el aborto provocado en el Congreso Nacional Brasileño: el papel del movimento de mujeres. Cadernos Saude Publica 1996;12(2):259-266.

11. Hardy E, Alves G. Complicações pós-aborto provocado: fatores associados. Cadernos Saude Publica 1992;8(4):454-458.

12. Passos MRL, Bravo RS. Planejamento familiar: abortamento. J Bras Medicina 1993;65(4):36-38.

13. Hofmeister VA. Estudo do término de gestações de trabalhadoras em indústria. Rev Bras Saude Ocup 1982;10(38): 38-42.

14. Boehs AE, Santos EK, Hasse M, Souza ML. Aborto provocado - estudo epidemiológico descritivo numa maternidade de Florianópolis, Santa Catarina. Ciencia Cultura (São Paulo) 1983;35(4):501-506.
15. Hardy EE, Costa RG, Rodrigues T, Moraes TM. Características atuais associadas a história de aborto provocado. Rev Saude Publica 1994;28(1):82-85.

16. Almeida MMG. Status da mulher e fertilidade. Rev Baiana Enfermagem 1994;7(1/2):22-33.

17. Hardy EE, Rebello I, Faúndes A. Aborto entre alunas e funcionárias de uma universidade brasileira. Rev Saude Publica 1993;27(2): 113-116.

18. Osis MJ, Hardy E, Faúndes A, Alves G, Balarezo G. Opinião das mulheres sobre as circunstâncias em que os hospitais deveriam fazer abortos. Cadernos Saude Publica 1994; 10(3):320-330.

19. Costa RG, Hardy E, Osis MJ, Faúndes A. A decisão de abortar: processo e sentimentos envolvidos. Cadernos Saude Publica 1995;11(1): 97-105.

20. León Aguirre DG, Salinas Urbina AA. Los médicos en formación y el aborto: opinión de estudiantes de medicina en la Ciudad de México. Cadernos Saude Publica 1997;13(2):227-235.
21. Tomasi E. As mães e suas gestações: comparação de duas coortes de base populacional no sul do Brasil. Cadernos Saude Publica 1996; 12(supl 1):21-25.

22. Gonzalez CH, Marques-Dias MJ, Kim CA, Sugayama SMM, Da Paz JA, Huson SM, Holmes LB. Congenital abnormalities in Brazilian children associated with misoprostol misuse in first trimester of pregnancy. Lancet 1998;351:1624-1627.

23. Osis MJD, Hardy EE, Faúndes A, Rodrigues T. Dificuldades para obter informações da população de mulheres sobre aborto ilegal. Rev Saúde Pública 1996;30(5):444-51.

24. Faúndes A, Cecatti JG, Bacha AM, Pinotti JA. Intervenções para a redução da mortalidade materna. Rev Paul Med 1989;107(1):47-52.

Manuscrito recebido em 30 de março de 1999. Aceito em versão revisada em 12 de dezembro de 1999.

ABSTRACT Abortion is not only a major cause of obstetric hospitalization in poor countries, but it also represents the failure of the public health system to provide enough information about contraceptive methods and thus prevent pregnancies. In Brazil, the high utilization rates of health facilities due to abortions reflect the ongoing difficulties with family planning and contraception. In addition, mortality resulting from abortions serves as an indicator of the quality of abortion procedures, an important point in a country where the practice is illegal and therefore done clandestinely. In this study, we analyzed the rates of mortality resulting from abortions among women 10 to 54 years old, including women who died from spontaneous and induced abortion, from 1980 to 1995, for the various regions of the country. The information we used came from the mortality data bank of the public health system of the Ministry of Health. Population data were obtained from the Brazilian Institute for Geography and Statistics. We studied 2602 deaths, 15\% of which were due to missed abortion, spontaneous abortion, or legally permitted induced abortion. The other $85 \%$ of the deaths were due to illegal induced abortions or to nonspecified abortions. The mortality rates from abortion-related causes have steadily decreased in all the regions of Brazil, but this improvement has been unevenly distributed in the country. The region with the smallest decrease in this rate ( $38 \%$ over 15 years) was the Northeast. The age of women dying from abortions progressively declined over the period studied. 